\title{
Nikfar Domination in Fuzzy Graphs
}

\author{
M. Nikfar \\ Independent Scholar \\ nikfarmath1@gmail.com
}

\begin{abstract}
The aim of this expository article is to present recent developments in the centuries-old discussion on the interrelations between several types of domination in graphs. However, the novelty even more prominent in the newly discovered simplified presentations of several older results. The main part of this article, concerning a new domination and older one, is presented in a narrative that answers two classical questions: (i) To what extend must closing set be dominating? (ii) How strong is the assumption of domination of a closing set? In a addition, we give an overview of the results concerning domination. The problem asks how small can a subset of vertices be and contain no edges or, more generally how can small a subset of vertices be and contain other ones. Our work was as elegant as it was unexpected being a departure from the tried and true methods of this theory that had dominated the field for one fifth a century. This expository article covers all previous definitions. The inability of previous definitions in solving even one case of real-world problems due to the lack of simultaneous attentions to the worthy both of vertices and edges causing us to make the new one. The concept of domination in a variety of graphs models such as crisp, weighted and fuzzy, has been in a spotlight. We turn our attention to sets of vertices in a fuzzy graph that are so close to all vertices, in a variety of ways, and study minimum such sets and their cardinality. A natural way to introduce and motivate our subject is to view it as a real-world problem. In its most elementary form, we consider the problem of reducing waste of time in transport planning. Our goal here is to first describe the previous definitions and the results, and then to provide an overview of the flows ideas in their articles. The final outcome of this article is twofold: (i) Solving the problem of reducing waste of time in transport planning at static state; (ii) Solving and having a gentle discussions on problem of reducing waste of time in transport planning at dynamic state. Finally, we discuss the results concerning holding domination that are independent of fuzzy graphs. We close with a list of currently open problems related to this subject. Most of our exposition assumes only familiarity with basic linear algebra, polynomials, fuzzy graph theory and graph theory.
\end{abstract}

Keywords : fuzzy graph, fuzzy bridge, $\alpha$-strong edge, nikfar domination, dynamic networks. AMS Subject Classification: 05C72, 05C69, 03E72, 94D05.

\section{Introduction and Overview}

Domination are among the most fundamental concepts of graph theory. Also, domination can behave in many strange ways. For instance, besides the classical definitions of domination, there are many characterization of this concept. One of this characterization due to A. Somasundaram and S. Somasundaram (Ref.[31]), see also Refs.[23, 14, 22, 13, 30, 15, 17, 24, 8, 32] for further generalizations. One the contrary and quite surprisingly, there are nowhere these definitions Solving the problem of reducing waste of time in transport planning and also (separately) all others real-world problems see 4. Somehow, a key direction of study of domination deals with trying to provide a clear structure of what the dominating set of vertices looks like. The leading theme of this expository article is to discuss the following two questions concerning fuzzy graphs

Q1: How much closing does dominating imply? 
Q2: How much dominating does closing imply?

They will be addressed in sections 3 and 4 , respectively. The main narrative presented in these sections is independent of any results from graph theory and/or calculus. The purpose of this expository article is to provide an overview of the authors' recent series of work (Refs. [31, 23, 14, 22, 13, 30, 15, 17, 24, 8, 32]), in which a positive answer to the problem of reducing waste of time in transport planning for the our new definition is given.

In 1965, Zadeh published his seminal paper "fuzzy sets" (Ref.[34]) as a way for representing uncertainty. In 1975, fuzzy graphs were introduced by Rosenfeld (Ref.[33]) and Yeh and Bang (Ref.[34]) independently as fuzzy models which can be used in problems handling uncertainty. Domination as a theoretical area in graph theory was formalized by Berge in 1958, in the chapter 4 with title " The fundamental Numbers of the theory of Graphs" (Ref.[1], Theorem 7, p.40) and Ore (Ref.[27], Chapter 13 , pp.206, 207) in 1962. Since 1977, when Cockayne and Hedetniemi (Ref.[7], Section 3, p.249-251) presented a survey of domination results, domination theory has received considerable attention. A set $S$ of vertices of $G$ (Ref.[5], Chap.10, p.302) is a dominating set if every vertex in $V(G)-S$ is adjacent to at least one vertex in $S$. The minimum cardinality among the dominating sets of $G$ is called the domination number of $G$ and is denoted by $\gamma(G)$. A dominating set of cardinality $\gamma(G)$ is then referred to as minimum dominationg set. Dominating sets appear to have their origins (Ref.[1], Example 2, p.41) in the game of chess, where the goal is to cover or dominate various squares of a chessboard by certain chess pieces. Consider a set of cities connected by communication paths, Which cities is connected to others by roads? We face with a graph model of this situation. But the cities are not same and they have different privileges in low traffic levels and this events also occur for the roads in low-cost levels. So we face with the weighted graph model, at first. These privileges are not crisp but they are vague in nature. So we don't have a weighted graph model. In other words, we face with a fuzzy graph model, which must study the concept of domination on it.

Next we turn our attention to sets of vertices in a fuzzy graph $G$ that are close to all vertices of $G$, in a variety of ways, and study minimum such sets and their cardinality.

In 1998, the concept of effective domination in fuzzy graphs was introduced by A. Somasundaram and S. Somasundaram (Ref. [31]) as the classical problems of covering chess board with minimum number of chess pieces. In 2010, the concept of 2-strong(weak) domination in fuzzy graphs was introduced by C. Natarajan and S.K. Ayyaswamy (Ref.[23]) as the extension of strong (weak) domination in crisp graphs. In 2014, the concept of 1-strong domination in fuzzy graphs was introduced by O.T. Manjusha and M.S. Sunitha (Ref.[14]) as the extension of domination in fuzzy graphs with strong edges. In 2015, the concept of 2-domination in fuzzy graphs was introduced by A. Nagoor Gani and K. Prasanna Devi (Ref.[22]) as the extension of 2-domination in crisp graphs. In 2015, the concept of strong domination in fuzzy graphs was introduced by O.T. Manjusha and M.S. Sunitha (Ref.[13]) as reduction of the value of old domination number and extraction of classic results. In 2016, the concept of $(1,2)$-domination in fuzzy graphs was introduced by N. Sarala and T. Kavitha (Ref.[30]) as the extension of $(1,2)$-domination in crisp graphs. A few researchers studied other domination variations which are based on above definitions, e.g. connected domination in Ref.([15], Definition 3.4, p.983), total domination in Ref.([17], Definition 3.4, p. 26), Independent domination in Ref.([24], Definition 3.5, p.16), Complementary nil domination in Ref.([8],Definition 3.1, p.3), Efficient domination in Ref.([32], Section 1, p. 9966). So we only compare our new definition with the fundamental dominations.

\section{Preliminaries}

We provide some basic background for the paper in this section.

We shall now list below some basic definitions and results of crisp graph, fuzzy subset and fuzzy graph from Refs.([5, 25, 20]), respectively.

We concern with a fuzzy graph which is defined on a crisp graph. So we recall the basic concepts of crisp graph. 
A graph (Ref.[5], p.1) $G^{*}$ is a finite nonempty set of objects called vertices (the singular is vertex) together with a (possibly empty) set of unordered pairs of distinct vertices of $G^{*}$ called edges. The vertex set of $G^{*}$ is denoted by $V\left(G^{*}\right)$, while the edge set is denoted by $E\left(G^{*}\right)$. Let $G^{*}=(V, E)$ be a finite graph. Then $G^{*}$ is called a simple graph, if it does not contain any loops or multiple edge at its vertices.

We recall that a fuzzy set in Ref.([25], Definition 1.2.1, p.3) of a set $S$ is a function of $S$ into the closed interval $[0,1]$.

We lay down the preliminary results which recall some basic concepts of fuzzy graph from Ref.[20].

Let $V$ be a nonempty finite set and $E \subseteq V \times V$. Then $G=(\sigma, \mu)$ is called a fuzzy graph on $V$, if $\forall u v \in E$, $\mu(u v)=\mu(v u) \leq \min \{\sigma(x), \sigma(y)\}$, where $\sigma: V \rightarrow[0,1]$ and $\mu: E \rightarrow[0,1]$ be the fuzzy set and $\mu$ is reflexive. The fuzzy graph $H=(\tau, \nu)$ is called a partial fuzzy subgraph of $G=(\sigma, \mu)$, if $\nu \subseteq \mu$ and $\tau \subseteq \sigma$. Similarly, the fuzzy graph $H=(\tau, \nu)$ is called a fuzzy subgraph of $G=(\sigma, \mu)$ on $V$ induced by $P$, if $P \subseteq V, \tau(x)=\sigma(x)$ for all $x \in P$ and $\nu(x y)=\mu(x y)$ for all $x, y \in P$. For the sake of simplicity, we sometimes call $H$ a fuzzy subgraph of $G$. We say that the partial fuzzy subgraph $(\tau, \nu)$ spans the fuzzy graph $(\sigma, \mu)$, if $\sigma=\tau$. In this case, we call $(\tau, \nu)$ a spanning fuzzy subgraph of $(\sigma, \mu)$.

A path $P$ of length $n$ is a sequence of distinct vertices $u_{0}, u_{1}, \cdots, u_{n}$ such that $\mu\left(u_{i-1} u_{i}\right)>0, i=$ $1,2, \cdots, n$ and $\min _{i=1,2, \cdots, n} \mu\left(u_{i-1} u_{i}\right)$ is defined as its strength. The strength of connectedness between two vertices $x$ and $y$ in $G$ is defined as the maximum of the strengths of all paths between $x$ and $y$ and is denoted by $\mu_{G}^{\infty}(x, y)$.

A fuzzy graph $G=(\sigma, \mu)$ on $V$ is connected if for every $x, y$ in $V, \mu_{G}^{\infty}(x, y)>0$.

Note that $\mu_{G^{\prime}}(x, y)$ is the strength of connectedness between $x$ and $y$ in the fuzzy graph obtained from $G$ by deleting the edge $x y$. An edge $x y$ in $G$ is $\alpha$-strong if $\mu(x y)>\mu_{G^{\prime}}(x, y)$. An edge $x y$ in $G$ is $\beta$-strong if $\mu(x y)=\mu_{G^{\prime}}(x, y)$. An edge $x y$ is a strong edge if it is either $\alpha$-strong or $\beta$-strong. An edge $u v$ of a fuzzy graph is called an $M$-strong edge, In order to avoid confusion with the notion of strong edges, we shall call strong in the sense of Mordeson as M-strong, if $\mu(u v)=\sigma(u) \wedge \sigma(v)$. If $\mu(u v)>0$, then $u$ and $v$ are called the neighbors. The set of all neighbors of $u$ is denoted by $N(u)$. Also $v$ is called the $\alpha$-strong neighbor of $u$, if the edge $u v$ is $\alpha$-strong. The set of all $\alpha$-strong neighbors of $u$ is denoted by $N_{s}(u)$. The degree of a vertex $v$ is defined as $d(v)=\Sigma_{u \neq v} \mu(u v)$. The $\alpha$-strong degree of a vertex $v \in V$ is defined as the sum of membership values of all $\alpha$-strong edges incident at $v$; It is denoted by $d_{s}(v)$; That is $d_{s}(v)=\Sigma_{u \in N_{s}(v)} \mu(u v)$. $v$ is called the effective neighbor of $u$, if the edge $u v$ is $M$-strong. The set of all $M$-strong neighbors of $u$ is denoted by $N_{e}(u)$. The $M$-strong degree of a vertex $v \in V$ is defined as the sum of membership values of all $M$-strong edges incident at $v$; It is denoted by $d_{e}(v)$; That is $d_{e}(v)=\Sigma_{u \in N_{e}(v)} \mu(u v)$.

A fuzzy graph $G=(\sigma, \mu)$ on $V$ is said complete in Ref.([20], Definition 2.3, p.28) if $\mu(u v)=\sigma(x) \wedge \sigma(y)$ for all $u, v \in V$.

The order $p$ and size $q$ of a fuzzy graph $G=(\sigma, \mu)$ on $V$ are defined $p=\Sigma_{x \in V} \sigma(x)$ and $q=\Sigma_{x, y \in V} \mu(x y)$. The scalar cardinality of $S$ is defined to be $\Sigma_{v \in S} \sigma(v)$ and it is denoted by $|S|_{s}$.

The complement of a fuzzy graph $G=(\sigma, \mu)$ on $V$, denoted by $\bar{G}$, is defined to $\bar{G}=(\sigma, \bar{\mu})$, where $\bar{\mu}(x y)=\sigma(x) \wedge \sigma(y)-\mu(x y)$ for all $x, y \in V$.

A fuzzy graph $G=(\sigma, \mu)$ on $V$ is said bipartite if the vertex set $V$ can be partitioned into two nonempty sets $V_{1}$ and $V_{2}$ such that $\mu\left(v_{1} v_{2}\right)=0$ if $v_{1}, v_{2} \in V_{1}$ or $v_{1}, v_{2} \in V_{2}$. Moreover, if $\mu(u v)=\sigma(u) \wedge \sigma(v)$ for all $u \in V_{1}$ and $v \in V_{2}$ then $G$ is called a complete bipartite fuzzy graph and is denoted by $K_{\sigma_{1}, \sigma_{2}}$, where $\sigma_{1}$ and $\sigma_{2}$ are respectively the restrictions of $\sigma$ to $V_{1}$ and $V_{2}$. In this case, If either $\left|V_{1}\right|=1$ or $\left|V_{2}\right|=1$ then the complete bipartite fuzzy graph is said a star fuzzy graph which is denoted by $K_{1, \sigma}$. A vertex $u$ is said isolated if $\mu(u v)=0$ for all $v \neq u$.

Now, we will define some special operations on fuzzy graphs. The pages of references will show the proof of validity of them.

The cartesian product in Ref.([19], Proposition 2.1, pp.160,161) $G=G_{1} \times G_{2}$ of two fuzzy graphs $G_{i}=\left(\sigma_{i}, \mu_{i}\right)$ on $V_{i}, i=1,2$ is defined as a fuzzy graph $G=\left(\sigma_{1} \times \sigma_{2}, \mu_{1} \times \mu_{2}\right)$ on $V \times V$ where $E=\left\{\left\{u u_{2}, u v_{2}\right\} \mid u \in V_{1}, u_{2} v_{2} \in E_{2}\right\} \cup\left\{\left\{u_{1} w, v_{1} w\right\} \mid u_{1} v_{1} \in E_{1}, w \in V_{2}\right\}$. Fuzzy sets $\sigma_{1} \times \sigma_{2}$ and $\mu_{1} \times \mu_{2}$ 
are defined as $\left(\sigma_{1} \times \sigma_{2}\right)\left(u_{1}, u_{2}\right)=\sigma_{1}\left(u_{1}\right) \wedge \sigma_{2}\left(u_{2}\right)$ and $\forall u \in V_{1}, \forall u_{2} v_{2} \in E_{2},\left(\mu_{1} \times \mu_{2}\right)\left(\left\{u u_{2}, u v_{2}\right\}\right)=$ $\sigma_{1}(u) \wedge \mu_{2}\left(u_{2} v_{2}\right)$ and $\forall u_{1} v_{1} \in E_{1}, \forall w \in V_{2},\left(\mu_{1} \times \mu_{2}\right)\left(\left\{u_{1} w, v_{w}\right\}\right)=\mu_{1}\left(u_{1} v_{1}\right) \wedge \sigma_{2}(w)$.

The union $G=G_{1} \cup G_{2}$ in Ref.([19], Proposition 3.1, pp.166,167) of two fuzzy graphs $G_{i}=\left(\sigma_{i}, \mu_{i}\right)$ on $V_{i}, i=1,2$ is defined as a fuzzy graph $G=\left(\sigma_{1} \cup \sigma_{2}, \mu_{1} \cup \mu_{2}\right)$ on $V_{1} \cup V_{2}$ where $E=E_{1} \cup E_{2}$. Fuzzy sets $\sigma_{1} \cup \sigma_{2}$ and $\mu_{1} \cup \mu_{2}$ are defined as $\left(\sigma_{1} \cup \sigma_{2}\right)(u)=\sigma_{1}(u)$ if $u \in V_{1}-V_{2},\left(\sigma_{1} \cup \sigma_{2}\right)(u)=\sigma_{2}(u)$ if $u \in V_{2}-V_{1}$, and $\left(\sigma_{1} \cup \sigma_{2}\right)(u)=\sigma_{1}(u) \vee \sigma_{2}(u)$ if $u \in V_{1} \cap V_{2}$. Also $\left(\mu_{1} \cup \mu_{2}\right)(u v)=\mu_{1}(u v)$ if $u v \in E_{1}-E_{2}$ and $\left(\mu_{1} \cup \mu_{2}\right)(u v)=\mu_{2}(u v)$ if $u v \in E_{2}-E_{1}$, and $\left(\mu_{1} \cup \mu_{2}\right)(u v)=\mu_{1}(u v) \vee \mu_{2}(u v)$ if $u v \in E_{1} \cap E_{2}$.

Let $G=G_{1}+G_{2}$ denote the join in Ref.([19], Proposition 3.3, p.168) of two fuzzy graphs $G_{i}=\left(\sigma_{i}, \mu_{i}\right)$ on $V_{i}, i=1,2$ is defined as a fuzzy graph $G=\left(\sigma_{1}+\sigma_{2}, \mu_{1}+\mu_{2}\right)$ on $V_{1} \cup V_{2}$ where $E=E_{1} \cup E_{2} \cup E^{\prime}$ and $E^{\prime}$ is the set of all edges joining vertices of $V_{1}$ with the vertices of $V_{2}$, and we assume that $V_{1} \cap V_{2}=\emptyset$. Fuzzy sets $\sigma_{1}+\sigma_{2}$ and $\mu_{1}+\mu_{2}$ are defined as $\left(\sigma_{1}+\sigma_{2}\right)(u)=\left(\sigma_{1} \cup \sigma_{2}\right)(u)$ and $\forall u \in V_{1} \cup V_{2} ;\left(\mu_{1}+\mu_{2}\right)(u v)=\left(\mu_{1} \cup \mu_{2}\right)(u v)$ if $u v \in E_{1} \cup E_{2}$ and $\left(\mu_{1}+\mu_{2}\right)(u v)=\sigma_{1}(u) \wedge \sigma_{2}(v)$ if $u v \in E^{\prime}$.

Definition 2.1. Let $G=(\sigma, \mu)$ be a fuzzy graph on $V$. Then

(i) (Ref.[31], Definition 2.9, p.3). $D \subseteq V$ is said to be effective dominating set, if for every $v \in V-D$, there exists $u$ in $D$ such that $\mu(u v)=\mu(u) \wedge \mu(v)$. Let $S$ be the set of all effective dominating sets in $G$. The effective domination number of $G$ is defined by $\gamma(G)=\min _{D \in S}\left(\Sigma_{u \in D} \sigma(u)\right)$.

(ii) (Ref.[23],p.1035). $D \subseteq V$ is said to be 2-strong(weak) dominating set, if for every $v \in V-D$, there exists $u$ in $D$ such that $\mu(u v)=\mu(u) \wedge \mu(v)$ and $d_{e}(u) \geq d_{e}(v)$. Let $S$ be the set of all 2-strong(weak) dominating sets in $G$. The 2-strong(weak) domination number of $G$ is defined by $\gamma_{s f}(G)\left(\gamma_{w f}(G)\right)=$ $\min _{D \in S}\left(\Sigma_{u \in D} \sigma(u)\right)$.

(iii) (Ref.[14], Definition 4.1(c), p.3208). $D \subseteq V$ is said to be 1-strong dominating set, if for every $v \in V-D$, there exists $u$ in $D$ such that $\mu(u v) \geq \mu_{G^{\prime}}^{\infty}(u, v)$. Let $S$ be the set of all 1-strong dominating sets in $G$. The 1-strong domination number of $G$ is defined by $\gamma_{S n}(G)=\min _{D \in S}\left(\Sigma_{u \in D} \sigma(u)\right)$.

(iv) (Ref.[22], Definition 3.1, p.120). $D \subseteq V$ is said to be 2-dominating set, if for every $v \in V-D$, there exists two vertices like $u$ in $D$ such that $\mu(u v)=\mu_{G^{\prime}}^{\infty}(u, v)$. Let $S$ be the set of all 2-dominating sets in the fuzzy graph $G$. Then The 2-domination number of $G$ is defined by $\gamma_{2}(G)=\min _{D \in S}\left(\Sigma_{u \in D} \sigma(u)\right)$.

(v) (Ref.[13], Definition 3.1, p.372). $D \subseteq V$ is said to be strong dominating set, if for every $v \in V-D$, there exists $u$ in $D$ such that $\mu(u v) \geq \mu_{G^{\prime}}^{\infty}(u, v)$. Let $S$ be the set of all strong dominating sets in $G$. The strong domination number of $G$ is defined by $\gamma_{s}(G)=\min _{D \in S}\left(\Sigma_{u \in D} t(u, v)\right)$ where $t(u, v)$ is the minimum of the membership values (weights) of the edge $u v$ such that $\mu(u v) \geq \mu_{G^{\prime}}^{\infty}(u, v)$.

(vi) (Ref.[30], Definition 3.1, p.16502). $D \subseteq V$ is said to be $(1,2)$-dominating set, if for every $v \in V-D$, there exists at least one vertex in $D$ at distance 1 from $v$ and a second vertex in $D$ at distance almost 2 from $v$. Let $S$ be the set of all $(1,2)$-dominating sets in $G$. The $(1,2)$-domination number of $G$ is defined by $\gamma_{(1,2)}(G)=\min _{D \in S}\left(\Sigma_{u \in D} \sigma(u)\right)$.

Remark 2.2. For the sake of simplicity, we do sometimes saying $\sigma(x)$ and $\mu(x y)$ with different literatures, e.g. value, weight, membership value and etc.

\section{New definition versus other ones: Restrictions, Extension Theorem and Monstrous examples}

Consider a set of cities connected by communication paths. Which cities have these properties? Having low traffic levels and other cities associating with at least ones by low-cost roads. We call this question as problem of reducing wast of time in transport planning. As outlined in Section 4, the previous definitions didn't consider values of vertices and edges, simultaneously. These parameters are simultaneously affected on any decision and analysis in transport planning. So those can't provide the appropriate solution to the problem. Therefore we decided to provide a new definition for the domination in fuzzy models.

To describe its generalization to fuzzy graph, it is helpful to reformulate this structure in three successive 
steps. The nikfar domination number of a fuzzy graph is defined in a classic way, (Definitions 3.1, 3.3 and 3.4) as reducing waste of time in transportation planning.

Definition 3.1. Let $G=(\sigma, \mu)$ be a fuzzy graph on $V$ and $x, y \in V$. We say that $x$ dominates $y$ in $G$ as $\alpha$-strong, if the edge $x y$ is $\alpha$-strong.

Example 3.2. Let $G=(\sigma, \mu)$ be a fuzzy graph as Figure 1. Then the edges $\left\{v_{2} v_{5}, v_{2} v_{4}, v_{3} v_{4}, v_{1} v_{3}\right\}$ are $\alpha$-strong and the edges $\left\{v_{1} v_{4}, v_{1} v_{2}, v_{4} v_{5}\right\}$ are not $\alpha$-strong.

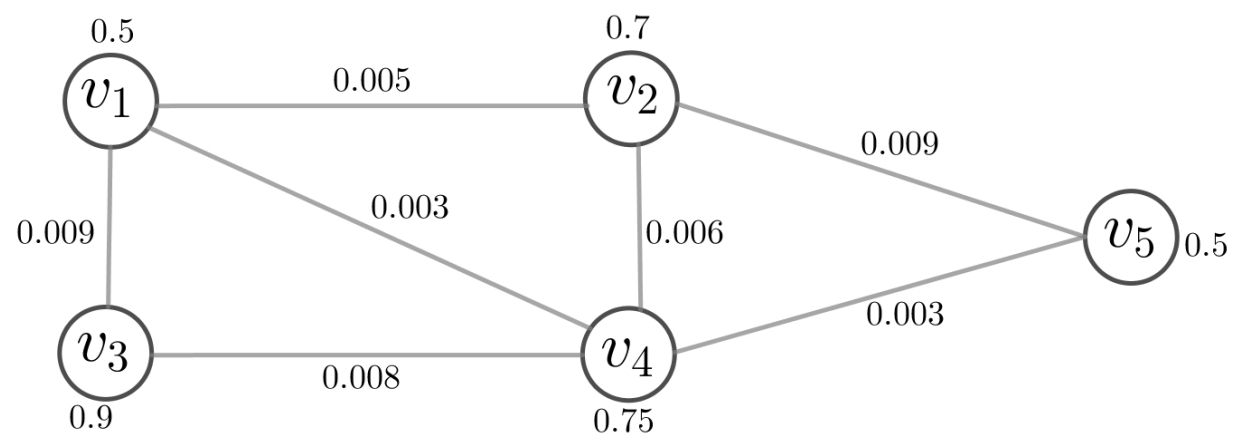

Figure 1: nikfar domination

Definition 3.3. Let $G=(\sigma, \mu)$ be a fuzzy graph on $V$. A subset $S$ of $V$ is called a $\alpha$-strong dominating set in $G$, if for every $v \in V-S$, there is $u \in S$ such that $u$ dominates $v$ as $\alpha$-strong.

Definition 3.4. Let $G=(\sigma, \mu)$ be a fuzzy graph on $V$. For every $u \in V$, the nikfar weight of $u$ is defined by $w_{v}(u)=\sigma(u)+\frac{d_{s}(u)}{d(u)}$. If $d(u)=0$, for some $u \in V$. Then we consider $\frac{d_{s}(u)}{d(u)}$ equal with 0 . For any $S \subseteq V$, natural extension of this concept to a set, is as follows. We also say the nikfar weight of $S$, it is defined by $w_{v}(S)=\Sigma_{u \in S}\left(w_{v}(u)\right)$. Now, let $U$ be the set of all $\alpha$-strong dominating sets in $G$. The nikfar domination number of $G$ is defined as $\gamma_{v}(G)=\min _{D \in U}\left(w_{v}(D)\right)$. The $\alpha$-strong dominating set that is correspond to $\gamma_{v}(G)$ is called by nikfar dominating set.

In what follows, we will work under this generality.

Example 3.5. Let $G=(\sigma, \mu)$ be a fuzzy graph as Figure 1. The set $S=\left\{v_{2}, v_{3}\right\}$ is an $\alpha$-strong dominating set. This set is also nikfar dominating set in fuzzy graph $G$. Hence $\gamma_{v}(G)=1.75+0.9+0.7=$ 3.35. So $\gamma_{v}(G)=3.35$.

Example 3.6. The following is a table consist of a brief fundamental comparison between types of domination in fuzzy graphs. There are two different types of the complete bipartite fuzzy graphs as Figures 2 and 3, which compare types of domination in fuzzy graphs.

\begin{tabular}{|c|c|c|c|}
\hline The types of edges & Types of Numbers & Figure 2 & Figure 3 \\
\hline$M-$ strong & Scalar cardinality & $\gamma(G)=1$ & $\gamma(G)=0.9$ \\
\hline$M-$ strong and $d_{e}(u) \geq d_{e}(v)$ & Scalar cardinality & $\gamma_{s f}(G)=1.2$ & $\gamma_{s f}(G)=1.1$ \\
\hline$M-$ strong and $d_{e}(u) \geq d_{e}(v)$ & Scalar cardinality & $\gamma_{w f}(G)=1$ & $\gamma_{w f}(G)=1.1$ \\
\hline strong & Scalar cardinality & $\gamma_{S n}(G)=0.9$ & $\gamma_{S n}(G)=1.6$ \\
\hline$\beta-$ strong & Scalar cardinality & $\gamma_{2}(G)=2.2$ & $\gamma_{2}(G)=1.6$ \\
\hline strong & $\Sigma_{u \in D} t(u, v)$ & $\gamma_{s}(G)=0.4$ & $\gamma_{s}(G)=0.2$ \\
\hline Distance & Scalar cardinality & $\gamma_{(1,2)}(G)=0.9$ & $\gamma_{(1,2)}(G)=0.9$ \\
\hline$\alpha-$ strong & nikfar weight & $\gamma_{v}(G)=2.2$ & $\gamma_{v}(G)=2.1$ \\
\hline
\end{tabular}




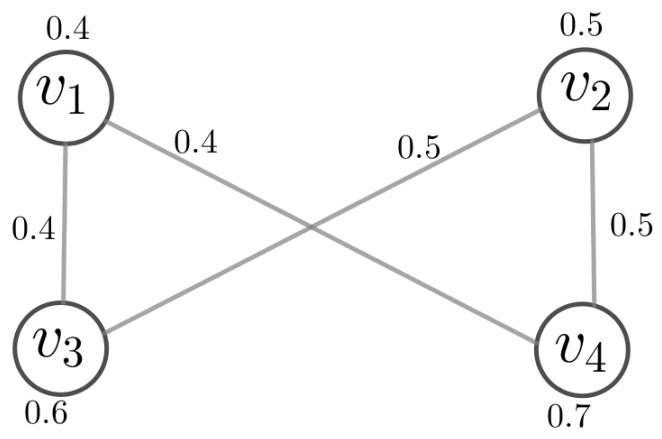

Figure 2: Comparison of dominations

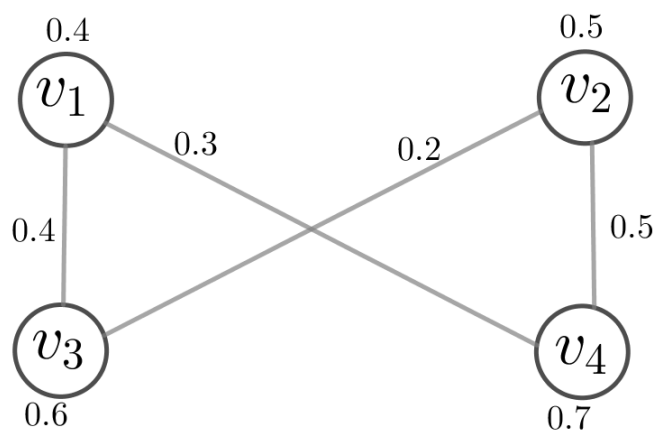

Figure 3: Comparison of dominations with different values

It is well known and generally accepted that the problem of determining the domination number of an arbitrary graph is a difficult one. Because of this, researchers have turned their attention to the study of classes of graphs for which the domination problem can be solved in polynomial time.

We determine nikfar domination number for several classes of fuzzy graphs consists of complete fuzzy graph, (Proposition 3.7), empty fuzzy graph, (Proposition 3.8), and complete bipartite fuzzy graph, (Proposition 3.10).

Proposition 3.7 (Complete fuzzy graph). Let $G=(\sigma, \mu)$ be a complete fuzzy graph on $V$ such that there is exactly one path with strength of $\mu^{\infty}(u, v)$. Then $\gamma_{v}(G)=\min _{u \in V}(\sigma(u))+1$.

Proof. Let $G$ be a complete fuzzy graph. The strength of path $P$ from $u$ to $v$ is of the form $\sigma(u) \wedge \cdots \wedge$ $\sigma(v) \leq \sigma(u) \wedge \sigma(v)=\mu(u v)$. So $\mu^{\infty}(u, v) \leq \mu(u v)$. uv is a path from $u$ to $v$ such that $\mu(u v)=\sigma(u) \wedge \sigma(v)$. Therefore $\mu^{\infty}(u, v) \geq \mu(u v)$. Hence $\mu^{\infty}(u, v)=\mu(u v)$. Then $\mu(u v)>\mu^{\prime}(u, v)$. It means that the edge $u v$ is $\alpha$-strong. All edges are $\alpha$-strong and each vertex is adjacent to all other vertices. So $D=\{u\}$ is a $\alpha$-strong dominating set and $d_{s}(u)=d(u)$ for each $u \in V$. The result follows.

Proposition 3.8 (Empty fuzzy graph). Let $G=(\sigma, \mu)$ be a edgeless fuzzy graph on $V$. Then $\gamma_{v}(G)=p$ where $p$ denotes the order of $G$.

Proof. $G$ is edgeless. Hence $V$ is only $\alpha$-strong dominating set in $G$ and there is no $\alpha$-strong edge. So by Definition 3.4, we have $\gamma_{v}(G)=\min _{D \in S}\left[\Sigma_{u \in D} \sigma(u)\right]=\Sigma_{u \in v} \sigma(u)=p$. Therefore $\gamma_{v}\left(\bar{K}_{n}\right)=p$ by our notations.

It is interesting to note the converse of Proposition 3.8, that does not hold. 
Example 3.9. We show that the converse of Proposition 3.8 does not hold. For this purpose, Let $V=\left\{v_{1}, v_{2}, v_{3}, v_{4}, v_{5}\right\}$. We define $\sigma$ on $V$ by $\sigma: V \rightarrow[0,1]$ such that

$$
\sigma\left(v_{1}\right)=0.5, \sigma\left(v_{2}\right)=0.7, \sigma\left(v_{3}\right)=0.9, \sigma\left(v_{4}\right)=0.75, \sigma\left(v_{5}\right)=0.5
$$

Now, The function $\mu: V \times V \rightarrow[0,1]$ is defined by $\mu\left(v_{1} v_{2}\right)=0.005$,

$$
\mu\left(v_{1} v_{4}\right)=0.003, \mu\left(v_{1} v_{3}\right)=0.009, \mu\left(v_{2} v_{4}\right)=0.006, \mu\left(v_{2} v_{5}\right)=0.009
$$

$\mu\left(v_{3} v_{4}\right)=0.008, \mu\left(v_{4} v_{5}\right)=0.003$ such that $\forall u, v \in V, \mu(u, v) \leq \sigma(u) \wedge \sigma(v)$. Finally, Let $V$, $\sigma$, and $\mu$ be the vertices, value of vertices and value of edges, respectively. The edges $\left\{v_{2} v_{5}, v_{2} v_{4}, v_{3} v_{4}, v_{1} v_{3}\right\}$ are $\alpha$-strong and the edges $\left\{v_{1} v_{4}, v_{1} v_{2}, v_{4} v_{5}\right\}$ are not $\alpha$-strong. So the set $\left\{v_{2}, v_{3}\right\}$ is the $\alpha$-strong dominating set. This set is also nikfar dominating set in fuzzy graph $G$. Hence $\gamma_{v}(G)=1.75+0.9+0.7=3.35=\Sigma_{u \in v} \sigma(u)=p$. Therefore $G \neq \bar{K}_{5}$ but $\gamma_{v}(G)=p$.

Proposition 3.10 (Complete bipartite fuzzy graph). Let $G=(\sigma, \mu)$ be the complete bipartite fuzzy graph on $V$ such that there is exactly one path with strength of $\mu^{\infty}(u, v)$. Then $\gamma_{v}(G)$ is either $\sigma(u)+1, u \in V$ or $\min _{u \in V_{1}, v \in V_{2}}(\sigma(u)+\sigma(v))+2$.

Proof. Let $G=(\sigma, \mu)$ be the complete bipartite fuzzy graph on $V$ such that there is exactly one path with strength of $\mu^{\infty}(u, v)$. By analogues to the proof of Theorem 3.7, all the edges are $\alpha$-strong.

If $G$ be the star fuzzy graph with $V=\left\{u, v_{1}, v_{2}, \cdots, v_{n}\right\}$ such that $u$ and $v_{i}$ are the center and the leaves of $G$, for $1 \leq i \leq n$, respectively. Then $\{u\}$ is the nikfar dominating set of $G$. Hence $\gamma_{v}(G)=\sigma(u)+1$.

Otherwise, both of $V_{1}$ and $V_{2}$ include more than one vertex. Every vertex in $V_{1}$ is dominated by every vertices in $V_{2}$, as $\alpha$-strong and conversely. Hence in $K_{\sigma_{1}, \sigma_{2}}$, the $\alpha$-strong dominating sets are $V_{1}$ and $V_{2}$ and any set containing 2 vertices, one in $V_{1}$ and other in $V_{2}$. So $\gamma_{v}\left(K_{\sigma_{1}, \sigma_{2}}\right)=\min _{u \in V_{1}, v \in V_{2}}(\sigma(u)+\sigma(v))+2$. The result follows.

Definition 3.11. (Ref.[20], Section 2.1, p.21) Let $G=(\sigma, \mu)$ be a fuzzy graph on $V$ and $x y \in E$. Then $x y$ is called a bridge if $\mu^{\prime} \infty(u, v)<\mu^{\infty}(u, v)$ for some $u, v \in V$, where $\mu^{\prime}(x y)=0$ and $\mu^{\prime}=\mu$ otherwise.

Theorem 3.12. (Ref.[20], Theorem 2.4, pp.21,22) Let $G=(\sigma, \mu)$ be a fuzzy graph on $V$ and $x y \in E$. Let $\mu^{\prime}$ be the fuzzy subset of $E$ such that $\mu^{\prime}(x y)=0$ and $\mu^{\prime}=\mu$ otherwise. Then $(3) \Leftrightarrow(2) \Leftrightarrow(1)$ :

(1) $x y$ is a bridge;

(2) $\mu^{\prime} \infty(x, y)<\mu(x y)$;

(3) $x y$ is not the weakest edge of any cycle.

Corollary 3.13. Let $G=(\sigma, \mu)$ be a fuzzy graph on $V$ and $x y \in E$. xy is an $\alpha$-strong edge if and only if $x y$ is a bridge.

Proof. By Theorem 3.12, the result is obviously hold.

Definition 3.14. (Ref.[20], Section 2.1, pp.22,23) A (crisp) graph that has no cycles is called acyclic or a forest. A connected forest is called a tree. A fuzzy graph is called a forest if the graph consisting of its nonzero edge is a forest and a tree if this graph is also connected. We call the fuzzy graph $G=(\sigma, \mu)$ a fuzzy forest if it has a partial fuzzy spanning subgraph which is a forest, where for all edges $x y$ not in $F[\mu(x y)=0]$, we have $\mu(x y)<\mu^{\infty}(x, y)$. In other words, if $x y$ is in $G$, but not $F$, there is a path in $F$ between $x$ and $y$ whose strength is greater than $\mu(x y)$. It is clear that a forest is a fuzzy forest. If $G$ is connected, then so is $F$ since any edge of a path in $G$ is either in $F$, or can be diverted through $F$. In this case, we call $G$ a fuzzy tree.

Theorem 3.15. (Ref.[20], Proposition 2.7, p.24) Let $G=(\sigma, \mu)$ be a fuzzy forest on $V$. Then the edges of $F=(\tau, \nu)$ are just the bridges of $G$. 
Corollary 3.16. Let $G=(\sigma, \mu)$ be a fuzzy forest on $V$. Then the edges of $F=(\tau, \nu)$ are just the $\alpha$-strong edges of $G$.

Proof. By Theorem 3.15 and Corollary 3.13, the result follows.

Proposition 3.17. Let $T=(\sigma, \mu)$ be a fuzzy tree on $V$. Then $D(T)=D(F) \cup D(S)$, where $D(T), D(F)$ and $D(S)$ are nikfar dominating sets of $T, F$ and $S$, respectively. $S$ is a set of edges which has no edges with connection to $F$.

Proof. By Corollary 3.16, the edges of $F=(\tau, \nu)$ are just the $\alpha$-strong edges of $G$. So by using Definition 3.4 , the result follows.

We give an upper bound for the nikfar domination number of fuzzy graphs, Proposition 3.18.

Proposition 3.18. For any fuzzy graph $G=(\sigma, \mu)$ on $V$, we have $\gamma_{v} \leq p$.

Proof. By Proposition 3.8, $\gamma_{v}\left(\bar{K}_{n}\right)=p$. So the result follows.

The classical paper in Ref.[26] of Nordhaus and Gaddum established the inequalities for the chromatic numbers of a graph $G=(V, E)$ and its complement $\bar{G}$. We are concerned with analogous inequalities involving domination parameters in graphs. We begin with a brief overview of Nordhaus-Gaddum (NG) inequalities for several domination-related parameters. For each generic invariant $\mu$ of a graph $G$, let $\mu=\mu(G)$ and $\bar{\mu}=\mu(\bar{G})$. Inequalities on $\mu+\bar{\mu}$ and $\mu \cdot \bar{\mu}$ exist in the literature for only a few of the many domination-related parameters and most of these results are of the additive form. In 1972 Jaeger and Payan (Ref.[9]) published the first NG results involving domination. Cockayne and Hedetniemi in Ref.[7] sharpened the upper bound for the sum. Laskar and Peters in Ref.[12] improved this bound for the case when both $G$ and $\bar{G}$ are connected. A much improved bound was established for the case when neither $G$ nor $\bar{G}$ has isolated vertices by Bollobás and Cockayne (Ref.[3]) and by Joseph and Arumugam (Ref.[10]) independently.

For any fuzzy graph the Nordhaus-Gaddum(NG)'s result holds, (Theorem 3.19).

Theorem 3.19. For any fuzzy graph $G=(\sigma, \mu)$ on $V$, the Nordhaus-Gaddum result holds. In other words, we have $\gamma_{v}+\overline{\gamma_{v}} \leq 2 p$.

Proof. Let $G$ be a fuzzy graph. So $\bar{G}$ is also fuzzy graph. We implement Theorem 3.18, on $G$ and $\bar{G}$. Then $\gamma_{v} \leq p$ and $\overline{\gamma_{v}} \leq p$. Hence $\gamma_{v}+\overline{\gamma_{v}} \leq 2 p$.

Definition 3.20. A $\alpha$-strong dominating set $D$ is called a minimal $\alpha$-strong dominating set if no proper subset of $\mathrm{D}$ is a $\alpha$-strong dominating set.

Theorem 3.21. Let $G=(\sigma, \mu)$ be a fuzzy graph without isolated vertices on $V$. If $D$ is a minimal $\alpha$-strong dominating set then $V-D$ is a $\alpha$-strong dominating set.

Proof. By attentions to all edges between two sets, which are only $\alpha$-strong, the result follows.

A domatic partition is a partition of the vertices of a graph into disjoint dominating sets. The maximum number of disjoint dominating sets in a domatic partition of a graph is called its domatic number.

Finding a domatic partition of size 1 is trivial and finding a domatic partition of size 2 (or establishing that none exists) is easy but finding a maximum-size domatic partition (i.e., the domatic number), is computationally hard. Finding domatic partition of size two in fuzzy graph $G$ of order $n \geq 2$ is easy by the following.

Theorem 3.22. Every connected fuzzy graph $G=(\sigma, \mu)$ of order $n \geq 2$ on $V$ has an $\alpha$-strong dominating set $D$ such that whose complement $V-D$ is also an $\alpha$-strong dominating set. 
Proof. For every connected fuzzy graph, $V$ is an $\alpha$-strong dominating set. By analogous to proof of Theorem 3.21, we can obtain the result.

We improve the upper bound for the nikfar domination number of fuzzy graphs without isolated vertices, (Theorem 3.23).

Theorem 3.23. For any fuzzy graph $G=(\sigma, \mu)$ without isolated vertices on $V$, we have $\gamma_{v} \leq \frac{p}{2}$.

Proof. Let $D$ be a minimal dominating set of $G$. By Theorem 3.22, V-D is an $\alpha$-strong dominating set of $G$. Hence $\gamma_{v}(G) \leq w_{v}(D)$ and $\gamma_{v}(G) \leq w_{v}(V-D)$.

Therefore $2 \gamma_{v}(G) \leq w_{v}(D)+w_{v}(V-D) \leq p$ which implies $\gamma_{v} \leq \frac{p}{2}$. Hence the proof is completed.

We also improve Nordhaus-Gaddum (NG)'s result for fuzzy graphs without isolated vertices, (Corollary 3.24).

Corollary 3.24. Let $G=(\sigma, \mu)$ be a fuzzy graph on $V$ such that both of $G$ and $\bar{G}$ have no isolated vertices. Then $\gamma_{v}+\overline{\gamma_{v}} \leq p$, where $\overline{\gamma_{v}}$ is the nikfar domination number of $\bar{G}$. Moreover, the equality holds if and only if $\gamma_{v}=\overline{\gamma_{v}}=\frac{p}{2}$.

Proof. By the Implement of Theorem 3.23, on $G$ and $\bar{G}$, we have $\gamma_{v}(G)=\gamma_{v} \leq \frac{p}{2}$, and $\gamma_{v}(\bar{G})=\overline{\gamma_{v}}(G)=$ $\overline{\gamma_{v}} \leq \frac{p}{2}$. So $\gamma_{v}+\bar{\gamma}_{v} \leq \frac{p}{2}+\frac{p}{2}=p$. Hence $\gamma_{v}+\overline{\gamma_{v}} \leq p$.

Suppose $\gamma_{v}=\overline{\gamma_{v}}=\frac{p}{2}$. Then obviously, $\gamma_{v}+\overline{\gamma_{v}}=p$. Conversely, suppose $\gamma_{v}+\overline{\gamma_{v}} \leq p$. Then we have $\gamma_{v} \leq \frac{p}{2}$ and $\overline{\gamma_{v}} \leq \frac{p}{2}$. If either $\gamma_{v}<\frac{p}{2}$ or $\overline{\gamma_{v}}<\frac{p}{2}$, then $\gamma_{v}+\overline{\gamma_{v}}<p$, which is a contradiction. Hence the only possible case is $\gamma_{v}=\overline{\gamma_{v}}=\frac{p}{2}$.

Proposition 3.25. Let $G=(\sigma, \mu)$ be a fuzzy graph on $V$. If all edges have equal value, then $G$ has no $\alpha$-strong edge.

Proof. By using Definition of $\alpha$-strong edge, the result is hold.

The following example illustrates this concept.

Example 3.26. In Figure 4, all edges have the same value but there is no $\alpha$-strong edges in this fuzzy graph.

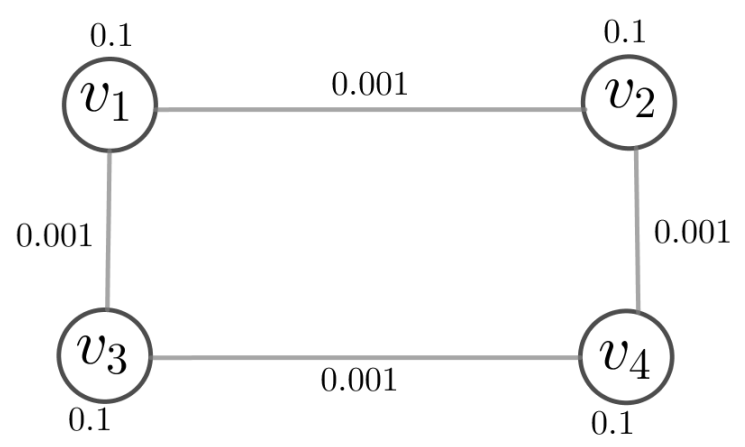

Figure 4: Identical edges and $\alpha$-strong edges

We give the relationship between $M$-strong edges and $\alpha$-strong edges, (Corollary 3.27).

Corollary 3.27. Let $G=(\sigma, \mu)$ be a fuzzy graph on $V$. If all edges are $M$-strong, then $G$ has no $\alpha$-strong edge.

Proof. By Proposition 3.25, the result follows. 
We give a necessary and sufficient condition for nikfar domination number which is half of order under the conditions. In fact, the fuzzy graphs which whose nikfar domination number is half of order, are characterized under the conditions, (Theorem 3.28).

Theorem 3.28. In any fuzzy graph $G=(\sigma, \mu)$ on $V$ such that values of vertices are equal and all edges have same value, i.e. $\forall u_{i}, u_{j} \in V$ and $\forall e_{i}, e_{j} \in E$, we have $\sigma\left(u_{i}\right)=\sigma\left(u_{j}\right)$ and $\mu\left(e_{i}\right)=\mu\left(e_{j}\right) \cdot \gamma_{v}=\frac{p}{2}$ if and only if for any nikfar dominating set $D$ in $G$, we have $|D|=\frac{n}{2}$.

Proof. Suppose $D$ has the conditions. By Proposition 3.25, $d_{s}(D)=0$. So by using Definition 3.4, $\gamma_{v}(G)=\Sigma_{u \in D} \sigma(u)$. Since values of vertices are equal and $|D|=\frac{n}{2}$, we have $\gamma_{v}(G)=\Sigma_{u \in D} \sigma(u)=$ $\frac{n}{2} \sigma(u)=\frac{1}{2}(n \sigma(u))=\frac{1}{2}\left(\Sigma_{u \in V} \sigma(u)\right)=\frac{1}{2}(p)=\frac{p}{2}$. Hence the result is hold in this case.

Conversely, suppose $\gamma_{v}=\frac{p}{2}$. Let $D=\left\{u_{1}, u_{2}, \cdots, u_{n}\right\}$ be a nikfar dominating set. By Proposition $3.25, d_{s}(D)=0$. So by using Definition 3.4, $\gamma_{v}(G)=\Sigma_{u \in D} \sigma(u)$. Since $\gamma_{v}(G)=W_{v}(D)$, we have $\gamma_{v}=$ $\frac{p}{2}=\frac{1}{2}\left(\Sigma_{u \in V} \sigma(u)\right)=\Sigma_{u \in D} \sigma(u)$. Suppose $n^{\prime} \neq \frac{n}{2}$. So $\left.\Sigma_{i=1}^{n^{\prime \prime}} \sigma\left(v_{i}\right)\right)=0$ which is a contradiction with $\forall u_{i} \in V, \sigma\left(u_{i}\right)>0$. Hence $n^{\prime}=\frac{n}{2}$, i.e. $|D|=n^{\prime}=\frac{n}{2}$. The result is hold in this case.

The nikfar domination of union of two fuzzy graphs is studied, (Proposition 3.29).

Proposition 3.29. Let $G_{1}$ and $G_{2}$ be fuzzy graphs. The nikfar dominating set of $G_{1} \cup G_{2}$ is $D=D_{1} \cup D_{2}$ such that $D_{1}$ and $D_{2}$ are the nikfar dominating sets of $G_{1}$ and $G_{2}$, respectively. Moreover, $\gamma_{v}\left(G_{1} \cup G_{2}\right)=$ $\gamma_{v}\left(G_{1}\right)+\gamma_{v}\left(G_{2}\right)$.

Proof. By using Definition of union of two fuzzy graphs, the result is obviously hold.

Also the nikfar domination of union of fuzzy graphs family is discussed, (Corollary 3.30).

Corollary 3.30. Let $G_{1}, G_{2}, \cdots, G_{n}$ be fuzzy graphs. The nikfar dominating set of $\cup_{i=1}^{n} G_{i}$ is $D=\cup_{i=1}^{n} D_{i}$ such that $D_{i}$ is the nikfar dominating set of $G_{i}$. Moreover, $\gamma_{v}\left(\cup_{i=1}^{n} G_{i}\right)=\sum_{i=1}^{n} \gamma_{v}\left(G_{i}\right)$.

Proof. By Proposition 3.29, the result is hold.

The concepts of both monotone increasing fuzzy graph property, (Definition 3.31), and monotone decreasing fuzzy graph property, (Definition 3.33), are introduced.

Definition 3.31. We call a fuzzy graph property $P$ monotone increasing if $G \in P$ implies $G+e \in P$, i.e., adding an edge e to a fuzzy graph $G$ does not destroy the property.

Example 3.32. Connectivity and Hamiltonicity are monotone increasing properties. A monotone increasing property is nontrivial if the empty fuzzy graph $\bar{K}_{\sigma} \notin P$ and the complete fuzzy graph $K_{\sigma} \in P$.

Definition 3.33. A fuzzy graph property is monotone decreasing if $G \in P$ implies $G-e \in P$, i.e., removing an edge from a graph does not destroy the property.

Example 3.34. Properties of a fuzzy graph not being connected or being planar are examples of monotone decreasing fuzzy graph properties.

Remark 3.35. Obviously, a fuzzy graph property $\mathrm{P}$ is monotone increasing if and only if its complement is monotone decreasing. Clearly not all fuzzy graph properties are monotone. For example having at least half of the vertices having a given fixed degree $d$ is not monotone.

In graph theory, Vizing's conjecture (Ref.[6]) concerns a relation between the domination number and the cartesian product of graphs. While the full conjecture remains open, Clark and Suen (2000) (Ref.[29]) have proved the looser result $\gamma(G) \gamma(H) \leq 2 \gamma(G \times H)$.

Vizing stated the still open conjecture:

Conjecture (Vizing Ref.[6]). For all graphs $G$ and $H, \gamma(G) \gamma(H) \leq \gamma(G \times H)$.

By using $\alpha$-strong edge and monotone decreasing fuzzy graph property, the result in relation with Vizing's conjecture is determined, (Theorem 3.36). 
Theorem 3.36. The Vizing's conjecture is monotone decreasing property in fuzzy graph $G$, if the edge e be $\alpha$-strong and $\gamma_{v}(G-e)=\gamma_{v}(G)$.

Proof. The fuzzy graph $(G-e) \times H$ is the spanning fuzzy subgraph of $G \times H$, for all fuzzy graph $H$. So $\gamma_{v}((G-e) \times H) \geq \gamma_{v}(G \times H) \geq \gamma_{v}(G) \gamma_{v}(H)=\gamma_{v}(G-e) \gamma_{v}(H)$. Hence Vizing's conjecture is also hold for $G-e$. Then the result follows.

By $\alpha$-strong edge and spanning fuzzy subgraph, some results in relation with Vizing's conjecture is studied, (Corollary 3.37).

Corollary 3.37. Suppose the Vizing's conjecture is hold for $G$. Let $K$ be the spanning fuzzy subgraph of $G$ such that $\gamma_{v}(K)=\gamma_{v}(G)$. Then the Vizing's conjecture is hold for $K$.

Proof. The fuzzy graph $K \times H$ is the spanning fuzzy subgraph of $G \times H$, for all fuzzy graph $H$. So $\gamma_{v}(K \times H) \geq \gamma_{v}(G \times H) \geq \gamma_{v}(G) \gamma_{v}(H)=\gamma_{v}(K) \gamma_{v}(H)$. Hence the Vizing's conjecture is also hold for $K$. So the result follows.

The nikfar domination of join of two fuzzy graphs is studied, (Proposition 3.38).

Proposition 3.38. Let $G_{1}$ and $G_{2}$ be fuzzy graphs. The nikfar dominating set of $G_{1}+G_{2}$ is $D=D_{1} \cup D_{2}$ such that $D_{1}$ and $D_{2}$ are the nikfar dominating set of $G_{1}$ and $G_{2}$, respectively. Moreover, $\gamma_{v}\left(G_{1}+G_{2}\right)=$ $\gamma_{v}\left(G_{1}\right)+\gamma_{v}\left(G_{2}\right)$.

Proof. By using Definition of join of two fuzzy graphs in this case, $M$-strong edges between two fuzzy graphs is not $\alpha$-strong which is a weak edge changing strength of connectedness of $G$.

Also the nikfar domination of join of fuzzy graphs family is discussed, (Corollary 3.39).

Corollary 3.39. Let $G_{1}, G_{2}, \cdots, G_{n}$ be fuzzy graphs. The nikfar dominating set of $+_{i=1}^{n} G_{i}$ is $D=$ $+_{i=1}^{n} D_{i}$ such that $D_{i}$ is the nikfar dominating set of $G_{i}$. Moreover, $\gamma_{v}\left(+_{i=1}^{n} G_{i}\right)=\sum_{i=1}^{n} \gamma_{v}\left(G_{i}\right)$.

Proof. By Proposition 3.38, the result is hold.

Gravier and Khelladi in Ref.[34] conjectured a Vizing-like inequality for the domination number of the join product of graphs; However, a counterexample was found by Klav žar Zmazek (1996) (Ref.[11]). For a more detailed overview of these results, see Brešar et al. (2012) (Ref.[4]).

Gravier and Khelladi stated the still open conjecture:

Conjecture (Gravier and Khelladi Ref.[34]). For all graphs $G$ and $H$,

$$
\gamma(G) \gamma(H) \leq 2 \gamma(G+H)
$$

By using $\alpha$-strong edge and monotone decreasing fuzzy graph property, the result in relation with the Gravier and Khelladi's conjecture is determined, (Theorem 3.40).

Theorem 3.40. The Gravier and Khelladi's conjecture is monotone decreasing property in fuzzy graph $G$, if the edge e be $\alpha$-strong and $\gamma_{v}(G-e)=\gamma_{v}(G)$.

Proof. The fuzzy graph $(G-e)+H$ is the spanning fuzzy subgraph of $G+H$, for all fuzzy graph $H$. So $2 \gamma_{v}((G-e)+H) \geq 2 \gamma_{v}(G+H) \geq \gamma_{v}(G) \gamma_{v}(H)=\gamma_{v}(G-e) \gamma_{v}(H)$. Hence the Gravier and Khelladi's conjecture is also hold for $G-e$. Then the result follows.

We conclude this section with some result in relation with the Gravier and Khelladi's conjecture, (Corollary 3.41).

Corollary 3.41. Suppose the Gravier and Khelladi's conjecture is hold for $G$. Let $K$ be the spanning fuzzy subgraph of $G$ such that $\gamma_{v}(K)=\gamma_{v}(G)$. Then the Gravier and Khelladi's conjecture is hold for $K$. 
Proof. The fuzzy graph $K+H$ is the spanning fuzzy subgraph of $G+H$, for all fuzzy graph $H$. So $2 \gamma_{v}(K+H) \geq 2 \gamma_{v}(G+H) \geq \gamma_{v}(G) \gamma_{v}(H)=\gamma_{v}(K) \gamma_{v}(H)$. Hence the Gravier and Khelladi's conjecture is also hold for $K$. The result follows.

\section{Practical Application}

In this section, we introduce one practical application in related to this concept. In the following, we will try to solve this problem by previous definitions. We show that these definitions are incapable of solving this problem and the new definition of this paper can give us a more realistic view of the situation and make it easier to understand the situation. In other words, this definition provides a solution to the problem that is consistent with reality. In the end, we will give a dynamic analysis of the status of this issue. In the dynamic state of this problem, we show that the previous definitions are even incapable of understanding the problem and we present dynamic and reality-based analysis by using the new definition.

Problem[reducing wast of time in transport planning] Consider a set of cities connected by communication paths. Which cities have these properties? Having low traffic levels and other cities associating with at least ones by low-cost roads.

The terms "low traffic" and "low-cost" are vague in nature. So we are faced with a fuzzy graph model. In other words, Let $G$ be a graph which represents the roads between cities. Let the vertices denote the cities and the edges denote the roads connecting the cities. From the statistical data that represents the high traffic flow of cities and high-cost roads, the membership functions $\sigma$ and $\mu$ on the vertex set and edge set of $G$ can be constructed by using the standard techniques given in Bobrowicz et al. in Ref.[2], Reha Civanlar and Joel Trussel Ref.[28]. In this fuzzy graph, a dominating set $S$ can be interpreted as a set of cities which have low traffic and every city not in $S$ is connected to a member in $S$ by a low-cost road. Suppose the Figure 5, the fuzzy graph model of the hypothetical condition of cities and the paths between them in a region. We now look at the answer to the problem raised by using the old and the

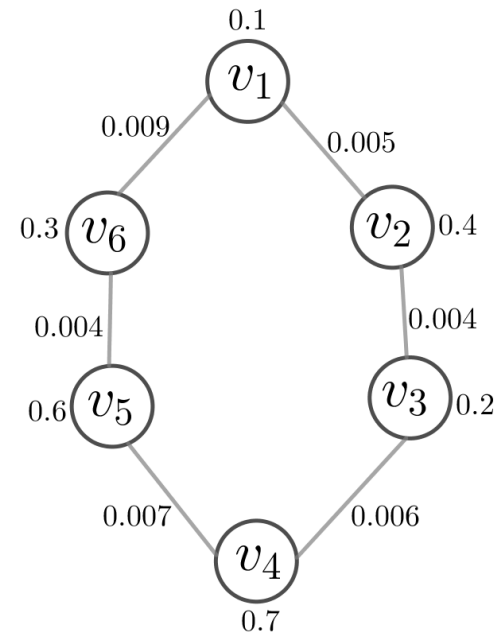

Figure 5: The exemplary scheme of road infrastructure

new definitions. As you can see in this model, finding the desirable cities is more important than finding the domination number. Because the numbers given for the set and each situation are compared with each others in the context of the same definition, and this number is merely to compare the different sets of cities in the context of the same definition. Therefore, speaking of the magnitude of this number in 
other definitions is meaningless. The table below illustrates the solutions presented for this problem.

\begin{tabular}{|c|c|}
\hline Definitions & Given desirable set \\
\hline A. Somasundaram and S. Somasundaram (Ref.[31]) & $V$ \\
\hline C. Natarajan and S.K. Ayyaswamy (Ref.[23]) & $V$ \\
\hline O.T. Manjusha and M.S. Sunitha (Ref.[14]) & $\left\{v_{3}, v_{6}\right\}$ \\
\hline A. Nagoor Gani and K. Prasanna Devi (Ref.[22]) & $V$ \\
\hline O.T. Manjusha and M.S. Sunitha (Ref.[13]) & $\left\{v_{3}, v_{6}\right\}$ \\
\hline N. Sarala and T. Kavitha (Ref.[30]) & $\left\{v_{3}, v_{6}\right\}$ \\
\hline Our new definition & $\left\{v_{1}, v_{4}\right\}$ \\
\hline
\end{tabular}

It is obvious from the above table and Figure 5 that the desirable cities given by previous definitions, are meaningless due to the lack of simultaneous attention to cities and roads. We are now presenting the dynamic status of the problem. The dynamic state is the situation in which the fuzzy graph model is found over time. Since over time, roads are becoming more affected and more precisely, they get worse, so the value of the roads increases, but cities do not change significantly over time, in their traffic. Because the traffic problem is an infrastructure problem. So in the Figure 6 presented by the dynamic fuzzy graph model, we present a situation in which, and over time, the value of the paths increases equally. In
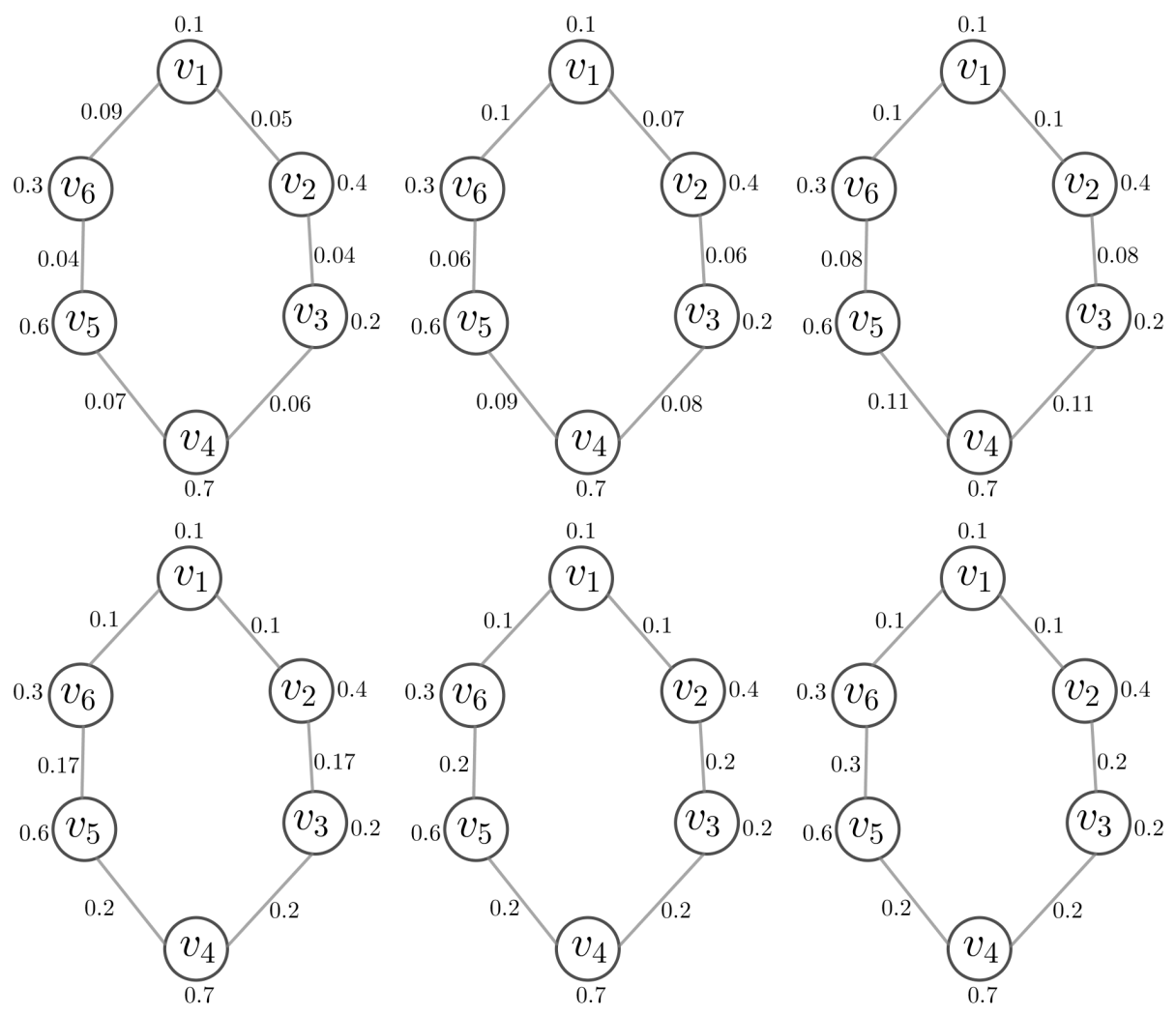

Figure 6: The dynamic scheme of road infrastructure

this situation, the answer given by the previous definitions reflects their inability to solve this problem, while the new definition adapts itself well to the new situation. The ineffectiveness and meaninglessness of previous definitions due to the lack of simultaneous attention to cities and roads. 
Dynamic analysis of networks in the first row of Figure 6 are the following table.

\begin{tabular}{|c|c|}
\hline Definitions & Given desirable set \\
\hline A. Somasundaram and S. Somasundaram (Ref.[31]) & $V, V-\left\{v_{6}\right\}, V-\left\{v_{2}, v_{6}\right\}$ \\
\hline C. Natarajan and S.K. Ayyaswamy (Ref.[23]) & $V, V-\left\{v_{6}\right\}, V-\left\{v_{2}, v_{6}\right\}$ \\
\hline O.T. Manjusha and M.S. Sunitha (Ref.[14]) & $\left\{v_{3}, v_{6}\right\},\left\{v_{3}, v_{6}\right\},\left\{v_{3}, v_{6}\right\}$ \\
\hline A. Nagoor Gani and K. Prasanna Devi (Ref.[22]) & $V, V, V$ \\
\hline O.T. Manjusha and M.S. Sunitha (Ref.[13]) & $\left\{v_{3}, v_{6}\right\},\left\{v_{3}, v_{6}\right\},\left\{v_{3}, v_{6}\right\}$ \\
\hline N. Sarala and T. Kavitha (Ref.[30]) & $\left\{v_{3}, v_{6}\right\},\left\{v_{3}, v_{6}\right\},\left\{v_{3}, v_{6}\right\}$ \\
\hline Our new definition & $\left\{v_{1}, v_{4}\right\},\left\{v_{1}, v_{4}\right\},\left\{v_{1}, v_{4}\right\}$ \\
\hline
\end{tabular}

According to the upper and lower tables, the desirable set given over time by using of the previous definitions, either provided the same solutions such as O.T. Manjusha and M.S. Sunitha (Ref.[14]), O.T. Manjusha and M.S. Sunitha (Ref.[13]) and N. Sarala and T. Kavitha (Ref.[30]) or in spite of a tangible change in their solutions to different situations, the general solutions have given. Additionally, the solutions of these definitions to the problem is not consistent with reality.

Dynamic analysis of networks in the second row of Figure 6 are the following table.

\begin{tabular}{|c|c|}
\hline Definitions & Given desirable set \\
\hline A. Somasundaram and S. Somasundaram (Ref.[31]) & $\left\{v_{1}, v_{4}\right\},\left\{v_{1}, v_{3}, v_{6}\right\},\left\{v_{3}, v_{6}\right\}$ \\
\hline C. Natarajan and S.K. Ayyaswamy (Ref.[23]) & $\left\{v_{1}, v_{4}\right\},\left\{v_{1}, v_{3}, v_{6}\right\},\left\{v_{3}, v_{6}\right\}$ \\
\hline O.T. Manjusha and M.S. Sunitha (Ref.[14]) & $\left\{v_{3}, v_{6}\right\},\left\{v_{3}, v_{6}\right\},\left\{v_{3}, v_{6}\right\}$ \\
\hline A. Nagoor Gani and K. Prasanna Devi (Ref.[22]) & $V-\left\{v_{1}\right\}, V-\left\{v_{1}\right\}, V-\left\{v_{1}\right\}$ \\
\hline O.T. Manjusha and M.S. Sunitha (Ref.[13]) & $\left\{v_{3}, v_{6}\right\},\left\{v_{3}, v_{6}\right\},\left\{v_{3}, v_{6}\right\}$ \\
\hline N. Sarala and T. Kavitha (Ref.[30]) & $\left\{v_{3}, v_{6}\right\},\left\{v_{3}, v_{6}\right\},\left\{v_{3}, v_{6}\right\}$ \\
\hline Our new definition & $\left\{v_{3}, v_{6}\right\},\left\{v_{3}, v_{6}\right\},\left\{v_{3}, v_{6}\right\}$ \\
\hline
\end{tabular}

\section{Conclusion}

The concept of domination in a variety of graphs models such as crisp, weighted and fuzzy graph models, has been in a spotlight. Due to the inability of previous definitions in solving the problem of reducing waste of time in transport planning due to the lack of simultaneous attention to cities and roads, we turn our attention to sets of vertices in a fuzzy graph $G$ that are close to all vertices of $G$, in a variety of ways, and study minimum such sets and their cardinality. We introduce a new variation on the domination theme, along with algebraic properties and mathematical results of it. This definition can give us a more realistic view of the situation and make it easier to understand the situation. In other words, this definition provides a solution to the problem that is consistent with reality. We also gave a dynamic analysis of the status of this problem. We hope these concepts are useful for studying problems of mathematics and real-world which make the future better as possible.

\section{Compliance with ethical standards}

Conflict of interest: The authors declare that there is no conflict of interest.

Human and animal rights: This article does not contain any studies with human participants or animals performed by any of the authors. 


\section{References}

[1] C. Berge, The Theory of Graphs and its Applications, Methuen, London, 1962.

[2] O. Bobrowicz, C. Choulet, A. Haurat, F. Sandoz, M. Tebaa, A method to build membership functionsApplication to numerical/symbolic interface building, in Proceedings of 3rd Internat. Conference on Information Processing and Management of Uncertainty in Knowledge Based System, Paris 1990.

[3] B. Bollobás and E.J. Cockayne, unpublished.

[4] Brešar, Boštjan; Dorbec, Paul; Goddard, Wayne; Hartnell, Bert L.; Henning, Michael A.; Klavžar, Sandi; Rall, Douglas F. (2012), Vizing's conjecture: a survey and recent results, Journal of Graph Theory 69 (1) 46-76.

[5] G. Chartrand and L. Lesniak, Graphs and Digraphs, Third edition, CRC Press, 1996.

[6] W.E. Clark and S. Suen, An Inequality Related to Vizing's Conjecture, Electronic J. Combinatorics $71(2000) 1-3,2000$.

[7] E.J. Cockayne and S.T. Hedetniemi, Toward a theory of domination in graphs, Networks 7 (1977) 247-261.

[8] M. Ismayil and I. Mohideen, Complementary nil domination in fuzzy graphs, Annals of Fuzzy Mathematics and Informatics (2014) 1-8.

[9] F. Jaeger and C. Payan, Relations du type Nordhaus-Gaddum pour le nombre d'absorption d'un graphe simple, C.R. Acad. Sci. Paris A 274 (1972) 728-730.

[10] J.P. Joseph and S. Arumugam, A note on domination in graphs, Internet. J. Management Systems, to appear.

[11] S. Klavžar, B. Zmazek, On a Vizing-like conjecture for direct product graphs, Discrete Mathematics, 156 (1996) 243-246.

[12] R. Laskar and K. Peters, Vertex and edge domination parameters in graphs, Congr. Numer. 48 (1985) 291-305.

[13] O.T. Manjusha and M.S. Sunitha, Strong Domination in Fuzzy Graphs, Fuzzy Inf. Eng. 7 (2015) 369-377.

[14] O.T. Manjusha and M.S. Sunitha, Notes on domination in fuzzy graphs, Journal of Intelligent and Fuzzy systems 27 (2014) 3205-3212.

[15] O.T. Manjusha and M.S. Sunitha, Connected domination in fuzzy graphs using strong edges, Annals of Fuzzy Mathematics and Informatics 10 (6) (2015) 979-994.

[16] O.T. Manjusha and M.S. Sunitha, The Strong Domination Alteration Sets in Fuzzy Graphs, International Journal of Mathematics and its Applications 4 (2-D) (2016) 109-123.

[17] O.T. Manjusha and M.S. Sunitha, Total Domination in Fuzzy Graphs Using Strong Edges, Annals of Pure and Applied Mathematics 9 (1) (2015) 23-33.

[18] S. Mathew and M.S. Sunitha, Types of edges in a fuzzy graph, Information Sciences 179 (2009) $1760-1768$.

[19] J.N. Mordeson and C.S. Peng, Operations on fuzzy graphs, Information Sciences 79 (1994) 159-170.

[20] J.N. Mordeson and P.S. Nair, Fuzzy Graphs and Fuzzy Hypergraphs, Physica-Verlag, 2000. 
[21] A. Nagoorgani and V.T. Chandrasekaran, Domination in fuzzy graph, Adv. in fuzzy sets and systems 1 (1) (2006) 17-26.

[22] A. Nagoor Gani and K. Prasanna Devi, 2-Domination in Fuzzy Graphs, International Journal of Fuzzy Mathematical Archive 9 (1) (2015) 119-124.

[23] C. Natarajan and S.K. Ayyaswamy, on Strong (weak) domination in Fuzzy Graphs, International Journal of Mathematical, Computational, Physical, Electrical and Computer Engineering 4 (7) (2010) 1035-1037.

[24] A. Nagoorgani and P.Vadivel, Relations between the parameters of Independent Domination and Irredundance in Fuzzy Graphs, International Journal of Algorithms, Computing and Mathmatics 2 (1) (2009) 15-19.

[25] H.T. Nguyen and E.A. Walker, A First course in fuzzy logic, CRC Press, 2006.

[26] E.A. Nordhaus and J.W. Gaddum, On complementary graphs, Amer. Math. Monthly 63 (1956) 175-177.

[27] O. Ore, The Theory of Graphs, Amer. Math. Soc. Colloq. Pub., providence, RI 381962.

[28] M. Reha Civanlar, H. Joel Trussel, Constructing membership functions using statistical data, Fuzzy Sets Syst.,18 (1986) 1-13.

[29] A. Rosenfeld, Fuzzy Graphs, In Fuzzy Sets and their Applications to Cognitive and Decision Processes, eds. L.A. Zadeh, K.S. Fu, K. Tanaka and M. Shimura, Academic Press, New York (1975) $77-95$.

[30] N. Sarala and T. Kavitha, (1,2)-Vertex Domination in Fuzzy Graphs, International Journal of Innovative Research in Science, Engineering and Technology 5 (7) (2016) 16501-16505.

[31] A. Somasundaram and S. Somasundaram, Domination in fuzzy graphs-I, Pattern Recognition Letters 19 (1998) 787-791.

[32] Dr.S. Vimala and J.S. Sathya, Efficient Domination number and Chromatic number of a Fuzzy Graph, International Journal of Innovative Research in Science, Engineering and Technology 3 (3) (2014)9965-9970.

[33] R.T. Yeh, S.Y. Bang, Fuzzy relations, fuzzy graphs and their applications to clustering analysis, in: Fuzzy Sets and Their Applications to Cognitive and Decision Processes, eds. L.A. Zadeh, K.S. Fu, K. Tanaka and M. Shimura, Academic Press, (1975) 125-149.

[34] L.A. Zadeh, Fuzzy sets, Information and Control 8 (1965) 338-353. 